\title{
Critical radius for hot-jet ignition of hydrogen-air mixtures
}

\author{
Jaime Carpio ${ }^{a}$, Immaculada Iglesias $^{b}$, Marcos Vera $^{b, *}$, Antonio L. Sánchez ${ }^{b}$, \\ Amable Liñán ${ }^{\mathrm{c}}$
}

Keywords:

Deflagration initiation

Hydrogen-air mixtures

Hot-jet ignition

Transient jets

\begin{abstract}
A B S T R A C T
This study addresses deflagration initiation of lean and stoichiometric hydrogen-air mixtures by the sudden discharge of a hot jet of their adiabatic combustion products. The objective is to compute the minimum jet radius required for ignition, a relevant quantity of interest for safety and technological applications. For sufficiently small discharge velocities, the numerical solution of the problem requires integration of the axisymmetric Navier-Stokes equations for chemically reacting ideal-gas mixtures, supplemented by standard descriptions of the molecular transport terms and a suitably reduced chemicalkinetic mechanism for the chemistry description. The computations provide the variation of the critical radius for hot-jet ignition with both the jet velocity and the equivalence ratio of the mixture, giving values that vary between a few tens microns to a few hundred microns in the range of conditions explored. For a given equivalence ratio, the critical radius is found to increase with increasing injection velocities, although the increase is only moderately large. On the other hand, for a given injection velocity, the smallest critical radius is found at stoichiometric conditions.
\end{abstract}

\section{Introduction}

It is well known that the combustion of a reactive mixture can be initiated by the sudden discharge of a hot jet of combustion products. This leads to the formation of a deflagration front when the jet radius $a$ is above a small critical value $a_{\mathrm{c}}$ on the order of the laminar flame thickness, whereas for smaller values of the jet radius the ignition kernel formed in the region where the hot products mix with the fresh reactants is too small to initiate a flame [1]. An analogy can be drawn to the problem of ignition by an external energy source, with the classical Spalding criterion for minimum ignition energy, according to which ignition is achieved when the size of the hot spot formed is comparable to or larger than the deflagration thickness, translating in the case of hot-jet ignition into the criterion for minimum jet radius mentioned above. This hotjet ignition phenomenon is important in connection with the handling and storage of gaseous fuels in the built environment, particularly hydrogen, where combustion transmission between adjacent enclosures emerges as a key issue. 
The problem has also technological applications of interest, an example being the hot-jet ignition systems investigated in Ref. [2], with applications to hydrogen-fired engines [3].

The problem of hot-jet ignition has been studied for hydrogen-air mixtures by a combination of experimental and numerical methods [4], providing increased understanding of the influence of the jet temperature and mixing process on ignition occurrence. On the other hand, influences of jet Reynolds number and limiting-reactant diffusivity on critical ignition conditions have been recently addressed by the authors in a computational work [5], which employed a one-step combustion reaction with Arrhenius kinetics along with a simplified flow field description with constant transport properties and constant density.

The starting jet formed by the sudden discharge of hot products into a quiescent atmosphere through a circular orifice in a plane wall includes a leading vortex ring followed by a slender jet stem, giving a flow field structure that has been studied in detail for constant density configurations [6,7] and also for lowMach-number gaseous jets with variable density [8]. The numerical results of Ref. [5] indicate that flame initiation is quite independent of the jet Reynolds number, whereas variations in the diffusivity of the limiting reactant are seen to have a larger effect, affecting in particular the ignition location. Thus, for values of the Lewis number of the order of or larger than unity, ignition tends to occur in the mixing layer surrounding the jet stem, while for very diffusive fuels with values of the Lewis number close to that of hydrogen, ignition occurs in the leading vortex ring, a phenomenon also observed in previous experimental studies [4]. For these small Lewis numbers differentialdiffusion effects were seen to lead to superadiabatic flame temperatures upon ignition, which greatly facilitate deflagration initiation when a temperature-sensitive reaction is considered [5].

Although the numerical results presented in our previous investigation [5] clarified a number of aspects concerning the deflagration initiation process, and identified key differential characteristics of very diffusive fuels, the quantitative results obtained cannot be directly applied to the evaluation of critical radii $a_{c}$ for ignition of hydrogen-air mixtures due to the simplifications introduced for the flow field and chemistry descriptions. Accurate quantitative predictions of $a_{c}$ require consideration of density variations along with an improved description of molecular transport and a reliable chemicalkinetic mechanism for the underlying chemistry. Such computations are reported below for hot-jet ignition of lean to stoichiometric hydrogen-air mixtures at normal atmospheric conditions, providing quantitative information of direct interest for safety applications.

\section{Numerical model}

A schematic representation of the configuration under study is presented in Fig. 1. An adiabatic flat wall separates a compartment containing a fresh mixture of hydrogen and air at normal atmospheric conditions, $p_{0}=1$ atm and $T_{0}=300 \mathrm{~K}$, from a compartment containing combustion products in equilibrium, with a temperature and composition corresponding to the adiabatic isobaric combustion of the fresh mixture found at the other side of the wall.

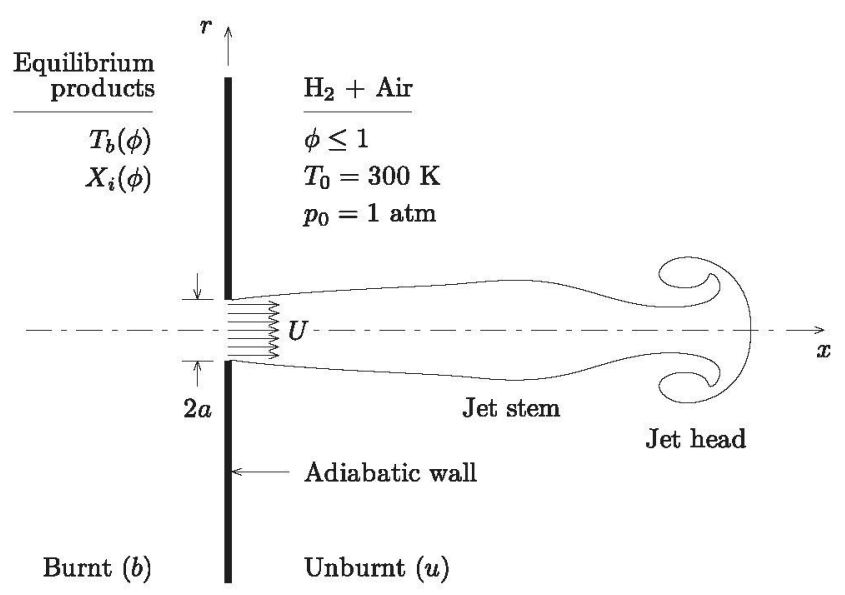

Fig. 1 - Schematic representation of the problem under study.

We shall consider that, at the initial instant, a jet of hot combustion products starts to discharge impulsively into the quiescent fresh mixture through a circular orifice of radius $a$. For simplicity in the calculations, a uniform constant value $U$ was used as entrance condition for the jet velocity across the orifice. This discharge velocity will be assumed to be small enough for compressibility effects to be unimportant, an accurate approximation when the overpressure in the products compartment is small compared with $p_{0}$. Since the critical radius lies always in the sub-millimeter range, the Reynolds numbers associated with these subsonic velocities are only moderately large, so that the resulting transient jets remain laminar and axisymmetric. Under these conditions, the numerical simulation of the transient jet discharge requires the integration of the axisymmetric Navier-Stokes equations for chemically reacting ideal-gas mixtures [1], that is, the conservation equations of momentum, energy and chemical species. A low-Mach-number approximation was employed when writing the energy equation and the equation of state. A three-step reduced chemical-kinetic mechanism involving $\mathrm{H}_{2}$, $\mathrm{O}_{2}, \mathrm{H}_{2} \mathrm{O}, \mathrm{HO}_{2}$, and $\mathrm{H}$ as reactive species, which has been tested to provide accurate results under a wide range of conditions including autoignition and deflagration propagation [9], was used for the chemistry description. This reduced mechanism has been recently shown to describe accurately autoignition of hydrogen in high-speed turbulent jets [10] and is therefore particularly suitable for the problem at hand. The governing equations are supplemented by the thermal and caloric equations of state, along with constitutive equations for the description of the molecular transport of momentum, energy, and species [1]. Mixture-averaged values are used for the thermodynamic properties and transport coefficients [11], with Soret diffusion included for $\mathrm{H}$ and $\mathrm{H}_{2}$, providing a standard transport description [12] analogous to that implemented in widely-used laminar combustion codes, such as Chemkin. Therefore, the present paper extends the previous numerical computations [5] by incorporating variable density, a sufficiently accurate model for molecular transport, and a reliable reduced mechanism for the chemistry description, all being necessary for the accurate quantification of ignition in hydrogen-air mixtures. 
The initial composition of the hydrogen-air mixture is uniquely determined by the equivalence ratio $\phi$, which also provides the thermochemical conditions in the resulting lowMach-number jet through an a-priori equilibrium calculation using the GASEQ code [13], as required to determine the adiabatic flame temperature $T_{b}$ and associated species mole fractions $\mathrm{X}_{i}$ of $\mathrm{H}_{2}, \mathrm{O}_{2}, \mathrm{H}_{2} \mathrm{O}, \mathrm{HO}_{2}$, and $\mathrm{H}$ in the product stream. Additional boundary conditions include an adiabatic impermeable wall together with an outflow condition for the velocity and pressure far from the orifice, where the composition and temperature are those of the unburnt mixture.

A space-adaptive finite-element semi-Lagrangian Runge-Kutta-Chebychev method, recently used e.g. in Ref. [14] for the description of unsteady diffusion flames, was employed for the numerical integration (further details of the numerical method can be found in Refs. [15-17]). The Navier-Stokes equations were integrated with a small constant time step $\Delta t^{\prime}=0.025 a / U$ in a cylindrical integration domain with radius $25 a$ and streamwise length 200a. Quadratic finite elements defined over a triangular mesh were used in the spatial discretization. The mesh, composed of elements of different sizes, was dynamically adapted to maintain a uniform distribution of the error at every instant of time. The smallest elements were located around the jet mixing layer, with a size on the order of a hundredth of the orifice radius, giving a total number of finite elements of the order of $10^{4}$ in cases without ignition, with this number increasing to approximately $3 \times 10^{4}$ when ignition appeared. Different time steps and also different element sizes were employed in sample computations to check the independence of the results with the selected discretization.

\section{Results of integration}

Besides the equivalence ratio $\phi$, corresponding to the ambient and jet conditions, the solution depends on the values of $a$ and $U$. For given values of $\phi$ and $U$, there exists a critical value of the orifice radius, $a_{c}$, above which a deflagration forms as a result of the jet discharge. To determine the parametric dependence $a_{c}(\phi, U)$, numerical integrations can be performed for increasing values of $a$ and fixed values of $\phi$ and $U$ until a successful ignition is observed. An alternative computation strategy, used in generating the results reported below, consists of keeping constant values of $\phi$ and Reynolds number $R e=\rho_{\mathrm{u}} U a / \mu_{\mathrm{u}}$ in the sequence of integrations for increasing values of $a$, with the Reynolds number defined with the density and viscosity of the unburnt mixture $\rho_{\mathrm{u}}$ and $\mu_{\mathrm{u}}$. The computations explored mixture compositions ranging from stoichiometric mixtures $(\phi=1)$ to near flammable lean conditions $(\phi=0.3)$ and injection conditions corresponding to Reynolds numbers in the range $250 \leq R e \leq 2000$.

Typical failed and successful ignition events are illustrated in Fig. 2, which displays temperature isocontours obtained at different times corresponding to $\phi=0.5, R e=1000$, and two different values of $a$. The dimensionless time $t=t^{\prime} /(a / U)$ is conveniently defined by scaling the time $t^{\prime}$ with the residence time $a / U$, whereas the axial and radial coordinates are scaled with the orifice radius $a$. The left-hand-side snapshots are computed for an orifice radius $a=0.184 \mathrm{~mm}$ below the critical
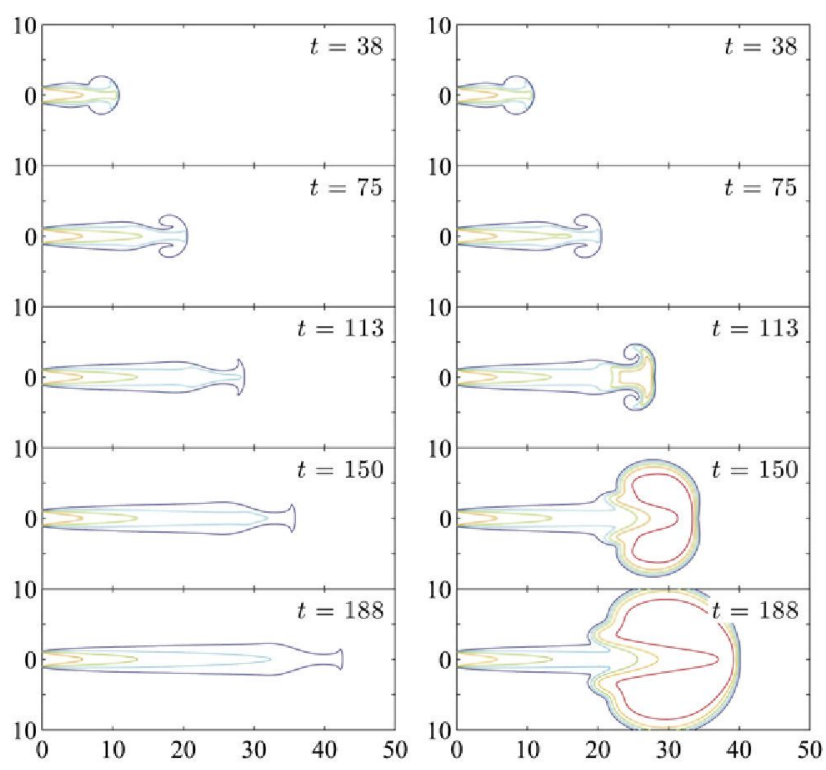

Fig. 2 - Failed and successful ignition events corresponding to $\phi=0.5$ and $\operatorname{Re}=1000$ for $a=0.184 \mathrm{~mm}$ (left) and $a=0.188 \mathrm{~mm}$ (right). The plots show temperature isocontours corresponding to $[2,3,4,5,6] \times 300 \mathrm{~K}$ for different times, scaled in the labels with the residence time $a / U$. The axial and radial coordinates are scaled with the orifice radius $a$.

value $a_{c}=0.186 \mathrm{~mm}$ corresponding to $\phi=0.5$ and $R e=1000$. As a result, the products in the jet mix with the ambient fresh mixture without significant chemical reaction. Note that due to the effect of heat conduction temperature differences in the jet head progressively smooth out as the jet develops, leaving behind a weakly-reactive flow that develops for large times into a slender steady jet.

The right-hand-side snapshots in Fig. 2 correspond to an orifice radius $a=0.188 \mathrm{~mm}>a_{\mathrm{c}}$. In the initial stages following the jet discharge, the hot products mix with the fresh mixture without appreciable chemical heat release, giving for $t=38$ a temperature distribution very similar to that found in the accompanying computation for $a<a_{\mathrm{c}}$. The effect of chemical reaction begins to modify the temperature field at later times, as can be seen in the plots for $t=75$ and, to a larger extent, for $t=113$, with a small ignition core identified behind the jet head in the former case that is soon convected downstream and engulfed by the leading vortex to form the incipient flame kernel observed for $t=113$. Combustion spreads from this kernel in the form of a relatively thick flame front that propagates in all directions, as indicated by the isocontours of temperature for $t=150$ and $t=188$, with downstream propagation being noticeably more rapid due to the predominant convective flow.

Results of critical ignition radii are summarized in Fig. 3, which shows the variation of $a_{c}$ with $U$ for different values of the equivalence ratio $\phi$. To help assess effects of molecular transport and compressibility, lines of constant Reynolds number Re and also of constant Mach number are included in the plot, with the Mach number $\mathrm{Ma}=\mathrm{U} / \mathrm{c}_{\mathrm{u}}$ defined with the ambient sound speed $c_{u}$, a quantity that depends in particular on the mixture composition. 


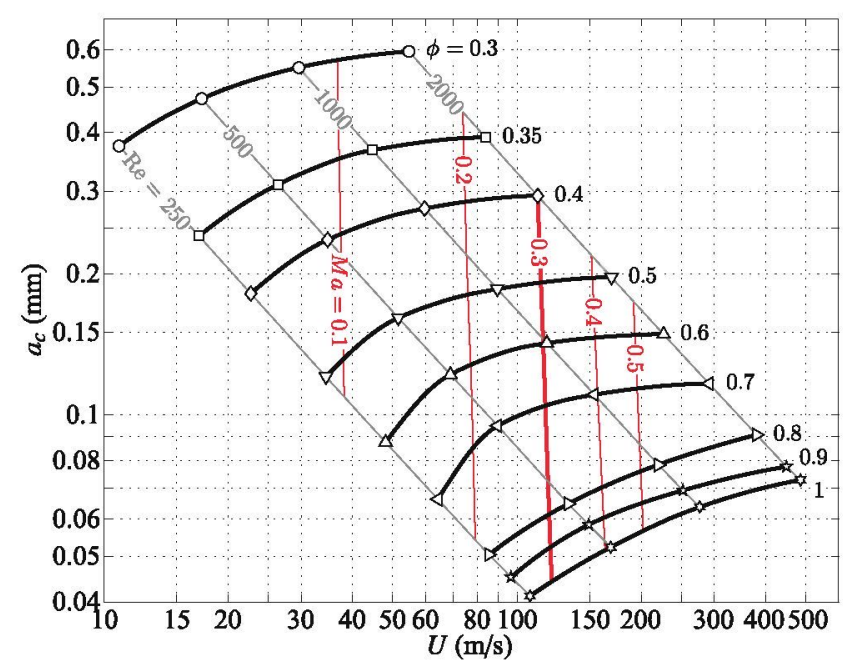

Fig. 3 - Variation of the jet critical radius $a_{\mathrm{c}}$ with the jet velocity $U$ for different values of the equivalence ratio $\phi$. Lines of constant Mach number $\mathrm{Ma}=\mathrm{U} / \mathrm{c}_{\mathrm{u}}$ and of constant Reynolds number $R e=\rho_{\mathrm{u}} \mathrm{U}_{\mathrm{a}} / \mu_{\mathrm{u}}$ are included for illustrative purposes.

As can be seen, for a given value of $\phi$, the ignition radius increases for increasing injection velocities. For instance, for $\phi=0.3$ the value of $a_{\mathrm{c}}$ increases by about $60 \%$ when the injection velocity increases from $U \simeq 11 \mathrm{~m} / \mathrm{s}$ to $U \simeq 55 \mathrm{~m} / \mathrm{s}$, corresponding to a variation of the associated jet Reynolds number from $\operatorname{Re}=250$ to $R e=2000$. This mild influence of the jet Reynolds number on the ignition process is in agreement with our previous work [5]. By way of contrast, variations of $a_{c}$ with $\phi$ are seen to be much more pronounced. For instance, for a stoichiometric mixture the resulting value of $a_{\mathrm{c}}$ for $R e=1000$ is only about $0.064 \mathrm{~mm}$, whereas for that same Reynolds number one obtains $a_{c}=0.550 \mathrm{~mm}$ for $\phi=0.3$.

This strong dependence on the composition is mostly a chemical-kinetic effect related to the progressive decrease of the jet temperature with decreasing values of $\phi$. To understand this, one should bear in mind that hydrogen ignition requires temperatures above a threshold value, the so-called crossover temperature [18], such that the chain-branching reactions can overcome the chain-terminating reactions to generate a net radical production rate. For the mixture to ignite, the jet temperature $T_{b}$ must be therefore above this value, on the order of $1000 \mathrm{~K}$ at atmospheric pressure. Since we assume that the jet of combustion products results from the adiabatic combustion of the fresh mixture, its temperature $T_{b}$ is just the adiabatic flame temperature for the value of $\phi$ selected in the computation. The equilibrium calculation provides a value of $T_{b}$ that decreases for decreasing values of $\phi$, reaching the crossover value when $\phi \simeq 0.25$, corresponding to the flammability limit of planar $\mathrm{H}_{2}$-air deflagrations $[19,20]$. For near-crossover conditions, the chemical reaction leading to ignition of the jet proceeds at a slow rate determined by the close competition of radical-branching and radicalrecombination reactions, so that ignition requires a larger residence time $a / U$, and therefore a larger value of $a$, thereby explaining the strong variation of $a_{c}$ with $\phi$ observed in Fig. 3 near the lean boundary. For the configuration selected, with the jet temperature being equal to the adiabatic flame temperature of the fresh mixture, no ignition may occur for values of $\phi<0.25$, so that the value of $a_{c}$ effectively diverges to infinity as the equivalence ratio approaches the chemicalkinetic limit $\phi=0.25$. Note, however, that hot-jet ignition of leaner mixtures could be in principle achieved by increasing the jet temperature to a sufficiently large value above crossover.

It is worth noting that compressibility effects, which were left out in our computations, are strictly negligible only for jets discharging with velocities $U$ much smaller than the ambient sound speed $c_{u}$, as occurs when the overpressure in the products container is much smaller than the ambient pressure $p_{0}$. In particular, the shock wave formed at the initial instant as the jet displaces suddenly the fresh mixture, which is merely an acoustic wave of negligibly small strength for $U /$ $c_{\mathrm{u}} \ll 1$, becomes stronger for larger values of $U / c_{\mathrm{u}}$, so that its presence can no longer be neglected when the jet velocity becomes comparable to the ambient sound speed, as occurs when the overpressure in the products container is of the order or larger than the ambient pressure $p_{0}$. Just like in other applications involving sudden jet discharge [21-23], this shock wave preheats the unburnt mixture as it propagates ahead of the jet, thereby facilitating ignition, although this effect is partly balanced by that of the accompanying expansion wave that propagates upstream from the orifice at the initial instant, cooling the hot products. These initial unsteady phenomena can be of particular importance for ignition near the leading edge of the head vortex, where one finds the reactive mixture that was shocked at the initial instant, when the shock-wave was strongest and the associated temperature jump was maximum. The counteracting effects of shock preheating of the reactive mixture and expansion cooling of the products would be more significant as $U / c_{u}$ increases, possibly exerting a significant influence for the conditions found in jet-ignition devices [3].

To ascertain whether these compressibility effects may play a role for the injection velocities corresponding to the results in Fig. 3, lines of constant Mach number $\mathrm{Ma}=\mathrm{U} / \mathrm{c}_{\mathrm{u}}$ are included in the plot. As can be seen, the results lying on the left-hand side of the plot correspond to incompressible jets with $\mathrm{Ma} \ll 1$, for which the computational approach taken here is strictly valid. This is no longer true for the points located on the right-hand side of the plot in the region where $M a \lesssim 1$. In the figure, the threshold value $M a=0.3$ is used to define an incompressible boundary, in that, for increased accuracy in the computation of $a_{c}$, compressibility effects should in principle be taken into account in the numerical simulations for conditions to the right of that boundary line.

\section{Conclusions}

Numerical integrations incorporating realistic descriptions for molecular transport and chemical kinetics have been used to determine the critical radius $a_{c}$ for ignition of a hydrogen-air mixture by a sudden axisymmetric jet discharge of its adiabatic combustion products. Atmospheric normal conditions $p_{0}=1 \mathrm{~atm}$ and $\mathrm{T}_{0}=300 \mathrm{~K}$ were selected for the mixture, as they are of direct interest for safety studies concerning the 
accidental ignition of hydrogen. The variation of $a_{c}$ with the injection velocity and equivalence ratio is calculated assuming negligible compressibility effects, giving the results summarized in Fig. 3, of direct interest for safety applications.

Larger values of the injection velocity and also larger values of the chamber pressure $p_{0}>1 \mathrm{~atm}$ and elevated initial temperatures $T_{0}>300 \mathrm{~K}$ could be of interest for other applications including jet-ignition devices for engines, for which compressibility effects are likely to be also relevant. Clearly, these issues are worth investigating in future numerical and experimental studies. Further work should also address the analysis of hydrogen-air mixtures by planar hot jets, which constitutes another configuration of interest for hydrogen safety applications.

\section{Acknowledgements}

This work was partially supported by Project S-505/ENE/0229 of the Spanish Comunidad de Madrid, and by projects CSD201000011 (CONSOLIDER-INGENIO) and MTM2010-18079 of the Spanish Ministerio de Economía y Competitividad.

\section{R E F E R E N G E S}

[1] Williams FA. Combustion theory. 2nd ed. Redwood City: Addison-Wesley; 1985.

[2] Murase E, Ono S, Hanada K, Oppenheim AK. Initiation of combustion in lean mixtures by flame jets. Combust Sci Technol 1996;113:167-77.

[3] Boretti AA, Watson HC. Enhanced combustion by jet ignition in a turbocharged cryogenic port fuel injected hydrogen engine. Int J Hydrogen Energy 2009;34:2511-6.

[4] Sadanandan R, Markus D, Schießl R, Maas U, Olofson J, Seyfried $\mathrm{H}$, et al. Detailed investigation of ignition by hot gas jets. Proc Combust Inst 2007;31:719-26.

[5] Iglesias I, Vera M, Sánchez AL, Liñán A. Numerical analyses of deflagration initiation by a hot jet. Combust Theory Model 2012;16:994-1010.

[6] Gharib M, Rambod E, Shariff K. A universal time scale for vortex ring formation. J Fluid Mech 1998;360:121-40.
[7] Rosenfeld M, Rambod E, Gharib M. Circulation and formation number of laminar vortex rings. J Fluid Mech 1998;376: 297-318.

[8] Iglesias I, Vera M, Sánchez AL, Liñán A. Simulations of starting gas jets at low Mach numbers. Phys Fluids 2005;17: 038105.

[9] Boivin P, Jiménez C, Sánchez AL, Williams FA. An explicit reduced mechanism for $\mathrm{H}_{2}$-air combustion. Proc Combust Inst 2011;33:517-23.

[10] Boivin P, Dauptain A, Jiménez C, Cuenot B. Simulation of a supersonic hydrogen-air autoignition-stabilized flame using reduced chemistry. Combust Flame 2012;159:1779-90.

[11] Kee RJ, Dixon-Lewis G, Warnatz J, Coltrin ME, Miller JA. A Fortran computer code package for the evaluation of gasphase, multicomponent transport properties. Albuquerque, NM: Sandia National Laboratories; 1986 . Report SAND 868246.

[12] Smooke MD. The computation of laminar flames. Proc Combust Inst 2012. http://dx.doi.org/10.1016/ j.proci.2012.09.005.

[13] Morley C. GASEQ: chemical equilibria in perfect gases, version 0.79, http://www.gaseq.co.uk/; Jan 2005.

[14] Carpio J, Sánchez-Sanz M, Fernández-Tarrazo E. Pinch-off in forced and non-forced, buoyant laminar jet diffusion flames. Combust Flame 2012;159:161-9.

[15] Bermejo R, Carpio J. An adaptive finite element semiLagrangian Runge-Kutta-Chebychev method for convection dominated reaction-diffusion problems. Appl Numer Math 2008;58:16-39.

[16] Bermejo R, Carpio J. A space-time element algorithm based on dual weighted residual methodology for parabolic equations. SIAM J Sci Comput 2009;31:3324-55.

[17] Bermejo R, Carpio J. A semi-Lagrangian-Galerkin projection scheme for convection equations. IMA J Numer Anal 2010;30: 799-831.

[18] Lewis B, Von Elbe G. Combustion, flames and explosions in gases. New York: Pergamon Press; 1951.

[19] Fernández-Galisteo D, Sánchez AL, Liñán A, Williams FA. One-step reduced kinetics for lean hydrogen-air deflagration. Combust Flame 2009;156:985-96.

[20] Fernández-Galisteo D, Sánchez AL, Liñán A, Williams FA. The hydrogen-air burning rate near the lean flammability limit. Combust Theory Model 2009;13:741-61.

[21] Dryer FL, Chaos M, Zhao Z, Stein JN, Alpert JY, Homer CJ. Spontaneous ignition of pressurized releases of hydrogen and natural gas into air. Combust Sci Technol 2007;179: 663-94.

[22] Mogi T, Wada Y, Ogata Y, Hayashi AK. Self-ignition and flame propagation of high-pressure hydrogen jet during sudden discharge from a pipe. Int J Hydrogen Energy 2009;34: $5810-6$.

[23] Bragin MV, Molkov VV. Physics of spontaneous ignition of high-pressure hydrogen release and transition to jet fire. Int J Hydrogen Energy 2011;36:2589-96. 